\title{
GENERALIZED REYNOLDS NUMBER FOR NON-NEWTONIAN FLUIDS
}

\author{
K. Madlener, B. Frey, and H. K. Ciezki
}

An extended version of the generalized Reynolds number was derived to characterize the duct flow of non-Newtonian gelled fluids of the HerschelBulkley-Extended (HBE) type. This number allows also estimating the transition from laminar to turbulent flow conditions. An experimental investigation was conducted with a capillary rheometer for several non-Newtonian gelled fluids to evaluate the introduced HBE-generalized Reynolds number $\mathrm{Re}_{\text {gen } \mathrm{HBE}}$. A good correlation between the experimental results and the theory could be found for laminar flow conditions. For one of the examined gelled fuels, the necessary high Reynolds numbers could be realized so that the transition from the laminar to the turbulent flow regime could be measured. Because of its general description, the HBE-generalized Reynolds number can also be applied to Newtonian liquids as well as to non-Newtonian fluids of the Herschel-Bulkley (HB), Ostwald-de-Waele (power-law, PL), and Bingham type.

\section{NOMENCLATURE}

$\begin{array}{ll}D & \text { duct diameter, m } \\ f_{\text {Darcy }} & \text { Darcy friction factor } \\ K & \text { prefactor of power-law, Pa·s }{ }^{n} \\ L & \text { duct length, m } \\ m & \text { local exponential factor } \\ n & \text { global exponential factor } \\ r & \text { radial coordinate, } \mathrm{m} \\ \mathrm{Re} & \text { Reynolds number } \\ \operatorname{Re}_{\text {Bingham }} & \text { Reynolds number for Bingham fluids } \\ \operatorname{Re}_{\mathrm{HBE}} & \text { Reynolds number for HBE-fluids } \\ \mathrm{Re}_{\mathrm{HB}} & \text { Reynolds number for HB-fluids } \\ \mathrm{Re}_{\mathrm{Newton}} & \text { Reynolds number for Newtonian fluids } \\ \operatorname{Re}_{\mathrm{PL}} & \text { Reynolds number for PL-fluids } \\ u(r) & \text { velocity, m/s } \\ \bar{u} & \text { average flow velocity, m/s }\end{array}$

This is an Open Access article distributed under the terms of the Creative Commons Attribution-Noncommercial License 3.0, which permits unrestricted use, distribution, and reproduction in any noncommercial medium, provided the original work is properly cited. 


\section{Greek letters}

$\begin{array}{ll}\dot{\gamma} & \text { shear rate, } \mathrm{s}^{-1} \\ \Delta p & \text { pressure loss, bar } \\ \eta & \text { dynamic shear viscosity, } \mathrm{Pa} \cdot \mathrm{s} \\ \rho & \text { density, } \mathrm{kg} / \mathrm{m}^{3} \\ \tau & \text { shear stress, } \mathrm{Pa} \\ \tau_{w} & \text { wall shear stress, } \mathrm{Pa} \\ \tau_{0} & \text { yield stress, } \mathrm{Pa}\end{array}$

\section{Abreviations and subscripts}

$\begin{array}{ll}\text { app } & \text { apparent } \\ \text { HB } & \text { Herschel-Bulkley } \\ \text { HBE } & \text { Herschel-Bulkley-extended } \\ \text { PL } & \text { power-law } \\ w & \text { wall }\end{array}$

\section{INTRODUCTION}

Gelled fuels and propellants are shear thinning non-Newtonian fluids with a significantly different viscosity behavior compared to Newtonian liquids [1]. Due to their safety and performance benefits, they are interesting candidates for rocket propulsion systems, see, e.g., Ciezki and Natan [2]. During storage and transport when very low shear forces occur, their viscosity is very high so that they are often described as semisolids. During the feeding process from the tank through the manifolds to the injector unit, high shear forces are applied to the fluids and their viscosity is strongly decreased. Thus, gelled propellants offer the possibility to build engines with thrust variation up to thrust cutoff and reignition like in engines with liquid propellants. At the same time, they have simple handling and storage characteristics like engines with solid propellants. Concerning the understanding of the duct flow characteristic of such non-Newtonian gelled fluids, there are still gaps to close. The present paper offers a further small step for a better understanding of the flow characteristics by defining an HBE-generalized Reynolds number.

To characterize or to compare the flow characteristics of fluids flowing through ducts, dimensionless numbers are often used. In 1883, Osborne Reynolds first introduced what is today known as the Reynolds number for fully developed duct flow of Newtonian liquids. The definition of the Newtonian Reynolds number $\mathrm{Re}_{\text {Newton }}$ is

$$
\operatorname{Re}_{\text {Newton }}=\frac{\rho D \bar{u}}{\eta_{\text {Newton }}}
$$




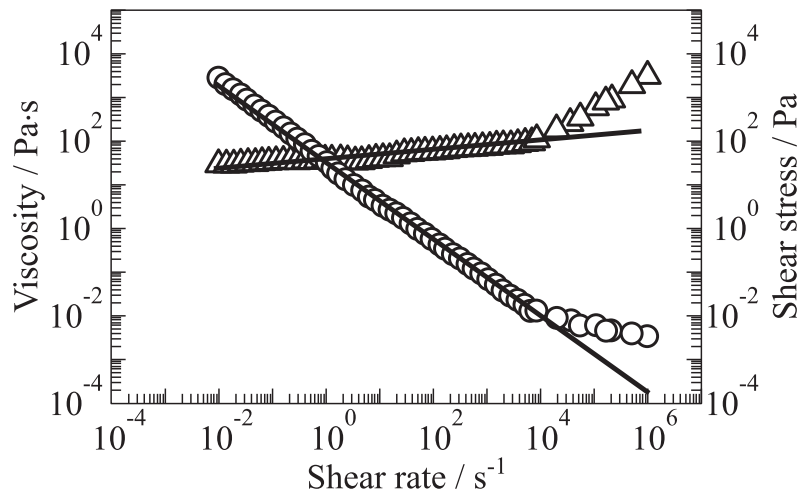

(a)

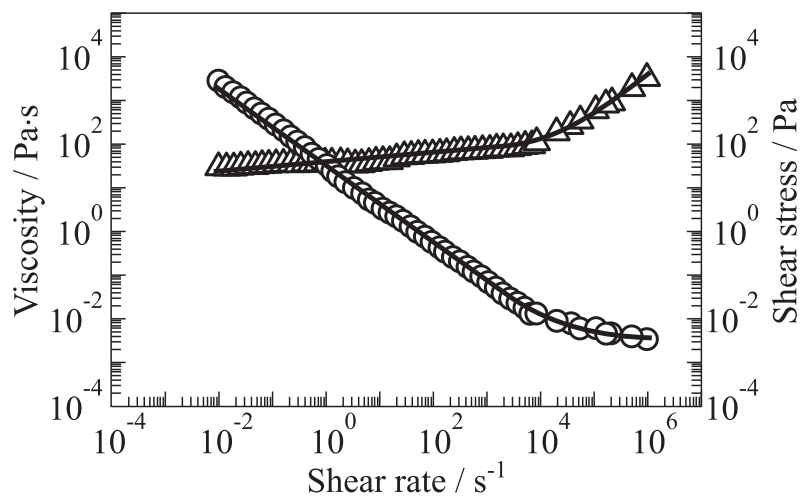

(b)

Figure 1 Viscosity measurements for a kerosene-thixatrol-gel and theoretical approach with PL $(a)$ and $\operatorname{HBE}(b): \triangle$ - viscosity and $\circ$ - shear stress

where $\rho$ is the fluid density, $D$ is the duct diameter, $\bar{u}$ is the average flow velocity, and $\eta_{\text {Newton }}$ is the constant Newtonian viscosity.

The Reynolds number can be interpreted as the ratio of inertial forces to viscous forces. It is commonly used to identify different flow regimes such as laminar or turbulent flow. Furthermore, it is used as a criterion for dynamic similitude that means, if two different flow configurations (different duct diameters, different flow rates, or different fluid properties) have the same dimensionless numbers, they are dynamically similar. As already mentioned, the Reynolds number

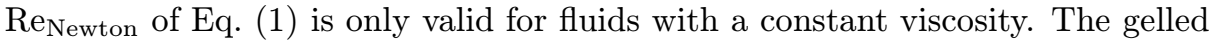
fluids investigated in the present paper, however, are non-Newtonian fluids with a more complex viscosity characteristic compared to Newtonian liquids. The diagrams in Fig. 1 show an example of the shear-rate dependent shear stress $\tau(\dot{\gamma})$, 
indicated with triangles, and the shear-rate dependent viscosity $\eta(\dot{\gamma})$, indicated with circles, for one of the investigated non-Newtonian fluids. The gel based on liquid kerosene was mixed in a Getzmann dissolver with $7.5 \%$ (wt.) Thixatrol ST and 7.5\%(wt.) 5-methyl-2-hexanon (miak). Rheological measurements were conducted with a Haake RS1 rotational rheometer (cone-plate) and a Rosand RH2000 capillary rheometer.

The duct flow of non-Newtonian fluids, particulary, for fluids with a viscosity characteristic following the Ostwald-de-Waele or PL equation $\eta_{\mathrm{PL}}=K \dot{\gamma}^{n-1}$, was investigated in the past, for example, by Dodge and Metzner [3], Ryan and Johnson [4], Mishra and Triphathi [5], Malin [6], and Böhme [7]. For the identification of different flow regimes or the determination of dynamic similitude, Metzner and Reed [8] introduced a generalized Reynolds number $\operatorname{Re}_{\text {gen PL valid }}$ for pure PL fluids. This number was derived from its relation to the Darcy friction factor $f_{\text {Darcy }}$ and is given by:

$$
\operatorname{Re}_{\text {gen PL }}=\frac{\rho D^{n} \bar{u}^{2-n}}{K((3 n+1) /(4 n))^{n} 8^{n-1}} .
$$

The fluids investigated in the present study, however, have a more complex viscosity characteristic that cannot be described by the PL all over the relevant shear rate range $10^{-2} \leq \dot{\gamma} \leq 10^{6} \mathrm{~s}^{-1}$. Figure $1 a$ shows the theoretical approach of the PL theory to the experimentally determined viscosity of the kerosene-gel. It can be seen that especially in the high shear-rate range, there is no good agreement between the theory and the experiments. The viscosity parameters for the PL-theory are $K=37.78 \mathrm{~Pa} \cdot \mathrm{s}$ and $n=0.12$ (fitted in the shear-rate range $\left.10^{1} \leq \dot{\gamma} \leq 10^{4} \mathrm{~s}^{-1}\right)$.

For better description of that viscosity characteristic, Madlener and Ciezki [9, 10] presented the HBE-equation as an extended version of the HB law. An additional viscosity term $\eta_{\infty}$ as the constant viscosity in the very high shearrate range was added to the term considering the existence of a yield stress $\tau_{0}$ and to the PL term $K \dot{\gamma}^{n-1}$. The definition of the HBE-theory is

$$
\begin{array}{r}
\eta_{\mathrm{HBE}}=\frac{\tau_{0}}{\dot{\gamma}}+K \dot{\gamma}^{n-1}+\eta_{\infty} \\
\text { with } \tau=\eta \dot{\gamma} \text { in general } \rightarrow \tau_{\mathrm{HBE}}=\tau_{0}+K \dot{\gamma}^{n}+\eta_{\infty} \dot{\gamma} .
\end{array}
$$

In Fig. $1 b$, the theoretical approach to the experimental viscosity data is shown for the HBE-law. Compared to the approach with the PL-theory in Fig $1 a$, the HBE-theory describes the experimental results over the entire relevant shear-rate range. The determined HBE-parameters for the kerosene-gel were $\tau_{0}=33 \mathrm{~Pa}, n=0.19, K=11.76 \mathrm{~Pa} \cdot \mathrm{s}^{0.19}$, and $\eta_{\infty}=0.0036 \mathrm{~Pa} \cdot \mathrm{s}$.

Since the PL theory is not capable of describing the viscosity characteristic of the fluids examined here over the entire relevant shear-rate range, the PL-based 


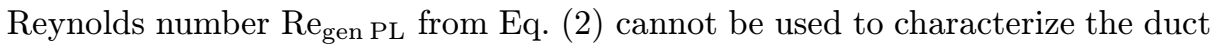
flow of such fluids either. For the characterization of flow and spray regimes of fluids following the HBE-viscosity type from Eq. (3), an HBE-generalized

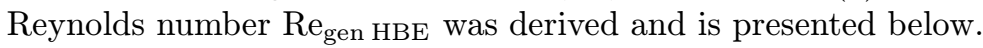

\section{HERSCHEL-BULKLEY-EXTENDED GENERALIZED REYNOLDS NUMBER}

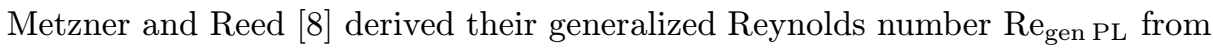
its relation to the Darcy friction factor. For laminar and fully developed duct flow, a relation exists between the Reynolds number and the Darcy friction factor which is generally given for fluids independent of their viscosity characteristic [11]. This relation reads

$$
\operatorname{Re}=\frac{64}{f_{\text {Darcy }}}
$$

whereas the definition of the Darcy friction factor is

$$
f_{\text {Darcy }}=\frac{(-\Delta p / L) D}{\rho \bar{u}^{2} / 2}
$$

where $\Delta p$ is the pressure drop over the duct length $L$, and $\bar{u}$ is the average flow velocity. The relation between the pressure loss and the wall shear stress $\tau_{w}$ is calculated by the equilibrium of forces over the duct with $\tau_{w}=(D /(4 L)) \Delta p$. The Darcy friction factor from Eq. (6) can then be written as

$$
f_{\text {Darcy }}=\frac{8 \tau_{w}}{\rho \bar{u}^{2}} .
$$

The wall shear stress $\tau_{w}$ in Eq. (7) can be calculated by the viscosity of the examined fluid. For a Newtonian liquid, there is a constant relation between the wall shear stress $\tau_{w}$ and the wall shear rate $\dot{\gamma}_{w}$ with $\tau_{w}=\eta \dot{\gamma}_{w}$, where $\eta$ is the constant Newtonian viscosity. The wall shear rate could then be calculated from the Newtonian velocity profile in duct flow with $\dot{\gamma}_{w}=8 \bar{u} / D$. With that, the Darcy friction factor would yield $f_{\text {Darcy }}=(64 \eta) /(\rho \bar{u} D)$ which gives Eq. (5). For a non-Newtonian fluid of the HBE-type, however, the wall shear stress $\tau_{w}$ has to be calculated by the appropriate viscosity law from Eq. (4).

Since the shear rate is defined as the negative gradient of the velocity profile $\dot{\gamma}=-d u / d r$, Eq. (4) yields the following expression if the velocity gradient is considered at the duct wall (denoted with index $w)$ :

$$
\tau_{w}=\tau_{0}+K\left(-\frac{d u}{d r}\right)_{w}^{n}+\eta_{\infty}\left(-\frac{d u}{d r}\right)_{w} .
$$


Rabinowitsch [12] and Mooney [13] developed an expression for the wall shear rate $\dot{\gamma}_{w}=-(d u / d r)_{w}$ independent of fluid properties and thus valid also for nonNewtonian fluids.

$$
\left(-\frac{d u}{d r}\right)_{w}=\frac{3}{4}\left(\frac{8 \bar{u}}{D}\right)+\frac{1}{4}\left(\frac{8 \bar{u}}{D}\right) \frac{d \ln (8 \bar{u} / D)}{d \ln (D \Delta p /(4 L))} .
$$

The expression $8 \bar{u} / D$ corresponds to the wall shear rate in case of Newtonian fluid flow and is named apparent wall shear rate $\dot{\gamma}_{\text {app } w}$. The expression $D \Delta p /(4 L)$ corresponds to the wall shear stress $\tau_{w}$. So, the logarithmic expression in Eq. (9) can be replaced with the reciprocal value of the local gradient $m$ which displays the gradient of the shear stress $\tau_{w}$ at a certain apparent wall shear rate $\dot{\gamma}_{\text {app } w}$ (Note: For a PL fluid, the local gradient $m$ would be identical with the global PL exponent $n$; for HBE-fluids, this is not the case.):

$$
\begin{aligned}
\frac{d \ln (8 \bar{u} / D)}{d \ln (D \Delta p /(4 L))} & =\frac{d \ln \left(\dot{\gamma}_{\text {app } w}\right)}{d \ln \left(\tau_{w}\right)}=\frac{1}{m} ; \\
\left(\frac{-d u}{d r}\right)_{w} & =\frac{3 m+1}{4 m} \frac{8 \bar{u}}{D} .
\end{aligned}
$$

Using Eqs. (8) and (10), the Darcy friction factor of Eq. (7) can be rewritten as follows:

$$
\begin{aligned}
& f_{\text {Darcy }}=8\left(\tau_{0}+\right.\left.K\left(\frac{3 m+1}{4 m}\right)^{n}\left(\frac{8 \bar{u}}{D}\right)^{n}+\eta_{\infty} \frac{3 m+1}{4 m} \frac{8 \bar{u}}{D}\right) /\left(\rho \bar{u}^{2}\right) \\
&=64\left(\frac{\tau_{0}}{8}\left(\frac{D}{\bar{u}}\right)^{n}+K\left(\frac{3 m+1}{4 m}\right)^{n} 8^{n-1}\right. \\
&\left.+\eta_{\infty} \frac{3 m+1}{4 m}\left(\frac{D}{\bar{u}}\right)^{n-1}\right) /\left(\rho \bar{u}^{2-n} D^{n}\right) .
\end{aligned}
$$

The generalized Reynolds number for laminar and fully developed duct flow valid for non-Newtonian fluids with a viscosity characteristic following the HBEequation can then be determined by inserting Eq. (11) in Eq. (5):

$$
\begin{aligned}
\operatorname{Re}_{\text {gen HBE }}=\left(\rho \bar{u}^{2-n} D^{n}\right) /\left(\frac{\tau_{0}}{8}\left(\frac{D}{\bar{u}}\right)^{n}+K\right. & \left(\frac{3 m+1}{4 m}\right)^{n} 8^{n-1} \\
& \left.+\eta_{\infty} \frac{3 m+1}{4 m}\left(\frac{D}{\bar{u}}\right)^{n-1}\right)
\end{aligned}
$$

with

$$
m=\frac{n K(8 \bar{u} / D)^{n}+\eta_{\infty}(8 \bar{u} / D)}{\tau_{0}+K(8 \bar{u} / D)^{n}+\eta_{\infty}(8 \bar{u} / D)}
$$


where $m$ is the local gradient of the shear stress vs. the shear rate in a log-log diagram. It was determined by the differentiation of the logarithmic expression for the HBE-equation according to:

$$
\begin{aligned}
& m=\frac{d \ln \left(\tau_{w}\right)}{d \ln \left(\dot{\gamma}_{\text {app } w}\right)}=\frac{d \ln \left(\tau_{0}+K \dot{\gamma}_{\text {app } w}^{n}+\eta_{\infty} \dot{\gamma}_{\text {app } w}\right)}{d \ln \left(\dot{\gamma}_{\text {app } w}\right)} \\
& m=\frac{d \ln \left(\tau_{0}+K e^{n \ln \left(\dot{\gamma}_{\text {app } w}\right)}+\eta_{\infty} e^{\ln \left(\dot{\gamma}_{\text {app } w}\right)}\right)}{d \ln \left(\dot{\gamma}_{\text {app } w}\right)} ; \\
& m=\frac{n K \dot{\gamma}_{\text {app } w}^{n}+\eta_{\infty} \dot{\gamma}_{\text {app } w}}{\tau_{0}+K \dot{\gamma}_{\text {app } w}^{n}+\eta_{\infty} \dot{\gamma}_{\text {app } w}}=\frac{n K(8 \bar{u} / D)^{n}+\eta_{\infty}(8 \bar{u} / D)}{\tau_{0}+K(8 \bar{u} / D)^{n}+\eta_{\infty}(8 \bar{u} / D)}
\end{aligned}
$$

where $\tau_{0}, K, n$, and $\eta_{\infty}$ are the HBE fluid parameters of the viscosity law from Eq. (3).

It is claimed that the introduced HBE-generalized Reynolds number $\mathrm{Re}_{\text {gen HBE }}$ from Eq. (12) is valid not only for fluids with a viscosity characteristic of the HBE-type but also for all viscosity laws included in that equation. Those are the HB, the Ostwald--de-Waele (PL), the Bingham, and the Newtonian laws. The reduced viscosity laws and their corresponding generalized Reynolds numbers are listed below:

- HBE:

$$
\eta=\frac{\tau_{0}}{\dot{\gamma}}+K \dot{\gamma}^{n-1}+\eta_{\infty} \longrightarrow \operatorname{Re}_{\text {gen HBE }}=\ldots \quad(\text { see Eq. }(12))
$$

- HB:

$$
\begin{aligned}
\eta_{\infty}=0 & \rightarrow \eta=\frac{\tau_{0}}{\dot{\gamma}}+K \dot{\gamma}^{n-1} \\
\longrightarrow \operatorname{Re}_{\text {gen HB }}=\frac{\rho \bar{u}^{2-n} D^{n}}{\left(\tau_{0} / 8\right)(D / \bar{u})^{n}+K((3 m+1) /(4 m))^{n} 8^{n-1}} & \text { with } m=\frac{n K(8 \bar{u} / D)^{n}}{\tau_{0}+K(8 \bar{u} / D)^{n}} ;
\end{aligned}
$$

- PL:

$$
\begin{aligned}
& \tau_{0}=0, \eta_{\infty}=0 \rightarrow \eta=K \dot{\gamma}^{n-1} \\
& \longrightarrow \operatorname{Re}_{\text {gen PL }}=\frac{\rho \bar{u}^{2-n} D^{n}}{K((3 m+1) /(4 m))^{n} 8^{n-1}} \\
& \text { with } m=n \text { (see Eq. (2)) } ;
\end{aligned}
$$


- Bingham:

$$
\begin{aligned}
& K=0, n=1 \rightarrow \eta=\frac{\tau_{0}}{\dot{\gamma}}+\eta_{\infty} \\
& \longrightarrow \operatorname{Re}_{\text {gen Bingham }}=\frac{\rho \bar{u} D}{\left(\tau_{0} / 8\right)(D / \bar{u})+\eta_{\infty}(3 m+1) /(4 m)} \\
& \text { with } m=\frac{\eta_{\infty}(8 \bar{u} / D)}{\tau_{0}+\eta_{\infty}(8 \bar{u} / D)}
\end{aligned}
$$

- Newton:

$$
\begin{aligned}
\tau_{0}=0, K=0, n=1 \rightarrow \eta=\eta_{\infty} \longrightarrow \operatorname{Re}_{\text {Newton }}=\frac{\rho \bar{u} D}{\eta_{\infty}} \\
\text { with } m=1 \text { (see Eq. (1)) . }
\end{aligned}
$$

It is worth mentioning that for a PL fluid, the HBE-generalized Reynolds number corresponds to the generalized Reynolds number by Metzner and Reed from Eq. (2). In case of a Newtonian liquid, the HBE-generalized Reynolds number reduces itself to the Newtonian Reynolds number from Eq. (1). Hence, the HBE-generalized Reynolds number can be applied to non-Newtonian fluids with the mentioned viscosity characteristics above, as well as to Newtonian liquids.

\section{VALIDATION AND EXPERIMENTAL RESULTS}

Experimental data are used for the evaluation of the introduced HBE-generalized Reynolds number $R_{\text {gen HBE. }}$. From the data, the Darcy friction factor $f_{\text {Darcy }}$ can be determined and plotted against the calculated Reynolds number. In the laminar flow region, the two parameters should follow the relation $f_{\text {Darcy }}$ $=64 /$ Re according to Eq. (5). The determination of the Darcy friction factor and the Reynolds number requires the information about the fluid properties, the duct geometry, and the measured pressure loss per volumetric flow rate. The test fluids (TF1-TF5) examined in this paper are two Newtonian liquids and three non-Newtonian gels with compositions according to Table 1 . The density of the kerosene-based fuels is about $\rho=800 \mathrm{~kg} / \mathrm{m}^{3}$, the density of the paraffin-based fuels is about $\rho=818 \mathrm{~kg} / \mathrm{m}^{3}$.

The viscosity behavior of the test fuels can be described by the HBEequation (3) over the entire relevant shear rate range $10^{-2} \leq \dot{\gamma} \leq 10^{6} \mathrm{~s}^{-1}$ relevant to injection processes. The corresponding fitted HBE-parameters are shown in Table 2.

The experiments for the evaluation were conducted with a Rosand RH2000 capillary rheometer. In the capillary rheometer, the examined fluid was driven 
Table 1 Composition of investigated test fuels in \%(wt.)

\begin{tabular}{lccc}
\hline \multicolumn{1}{c}{ Test fluid } & Basic fuel & Gellant & Additive \\
\hline TF1 (Newtonian) & 100\% paraffin & - & - \\
TF2 (Newtonian) & 100\% kerosene & - & - \\
TF3 (non-Newtonian) & $85 \%$ paraffin & $7.5 \%$ thixatrol ST & $7.5 \%$ miak \\
TF4 (non-Newtonian) & $96 \%$ paraffin & $4.0 \%$ aerosil 200 & - \\
TF5 (non-Newtonian) & $85 \%$ kerosene & $7.5 \%$ thixatrol ST & $7.5 \%$ miak \\
\hline
\end{tabular}

Table 2 Herschel-Bulkley-extended parameters of the test fuels TF1-TF5

\begin{tabular}{ccccc}
\hline Test fluid & $\tau_{0}, \mathrm{~Pa}$ & $n$ & $K, \mathrm{~Pa} \cdot \mathrm{s}^{n}$ & $\eta_{\infty}, \mathrm{Pa} \cdot \mathrm{s}$ \\
\hline TF1 & - & 1 & - & 0.026 \\
TF2 & - & 1 & - & 0.0012 \\
TF3 & 45 & 0.38 & 5.07 & 0.026 \\
TF4 & 83 & 0.57 & 2.54 & 0.026 \\
TF5 & 33 & 0.19 & 11.76 & 0.0036 \\
\hline
\end{tabular}

by a piston from the reservoir through a capillary. Pressure losses were messured at several volumetric flow rates with capillaries of diameters $D=0.2$ and $0.3 \mathrm{~mm}$ and lengths $L=16$ and $24 \mathrm{~mm}$. The necessary corrections were applied to the raw data $[12,14]$.

To demonstrate the advantage of the introduced generalized Reynolds number $\operatorname{Re}_{\text {gen HBE }}$ in comparison to the Newtonian Reynolds number Re $\mathrm{N}_{\text {Newton }}$ and the generalized number for PL fluids $R_{\text {gen } P L}$, the experimental data for the kerosene-thixatrol-gel (TF5) are shown as an example in three diagrams of Fig. 2.

In the diagrams, the Darcy friction factor is plotted vs. the Reynolds number of the Newtonian, PL, and HBE-definitions. The viscosity value for calculating the Newtonian Reynolds numbers (Eq. (1)) in Fig. $2 a$ was chosen with $\eta_{\text {Newton }}$ $=0.0036 \mathrm{~Pa} \cdot \mathrm{s}$ as the viscosity value of the gel in the very high shear rate range. The PL parameters for calculating the PL Reynolds number (Eq. (2)) in Fig. $2 b$ were determined with $K=37.78 \mathrm{~Pa} \cdot \mathrm{s}$ and $n=0.12$ according to the PL fit in Fig. 1. The HBE-parameters for calculating the HBE-Reynolds number (Eq. (12)) in Fig. $2 c$ were taken from Table 2.

In all three diagrams, the solid line corresponds to the ratio $f_{\text {Darcy }}=64 /$ Re for laminar flow, which is generally given for fluids independent of their viscosity characteristic. It can be seen that the experimental data of the friction factor values comply with this relation only when plotted against the generalized HBEReynolds number $\mathrm{Re}_{\text {gen HBE}}$. The calculation of the Reynolds number with the Newtonian or the PL definitions yields significant errors. For high Reynolds 


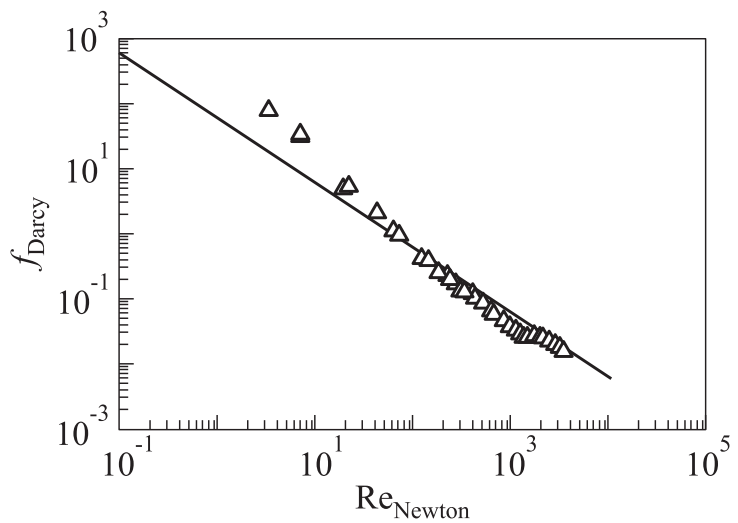

(a)

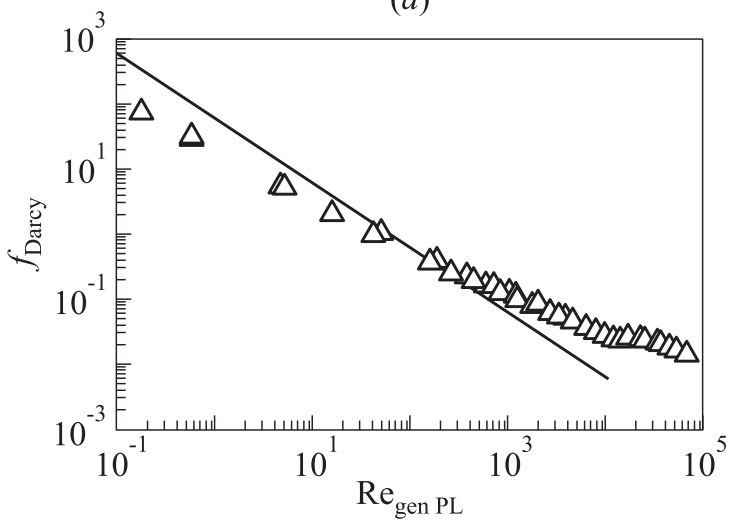

(b)

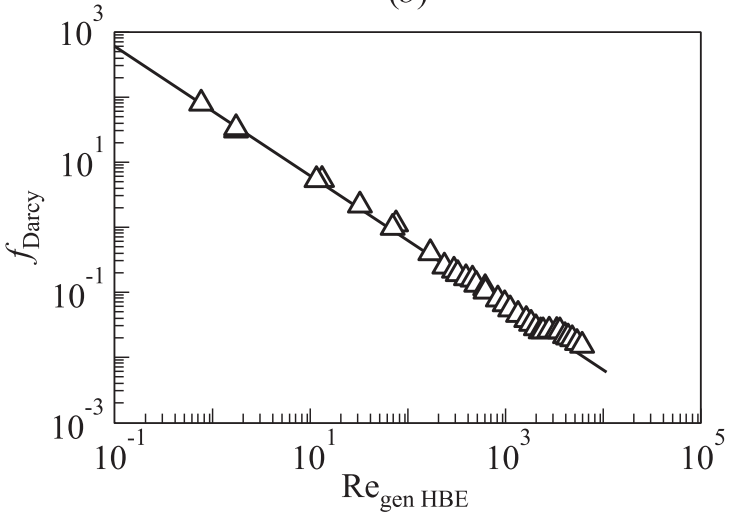

(c)

Figure 2 Friction factor vs. different definitions of the Reynolds number for the kerosene-thixatrol-gel (TF5) 


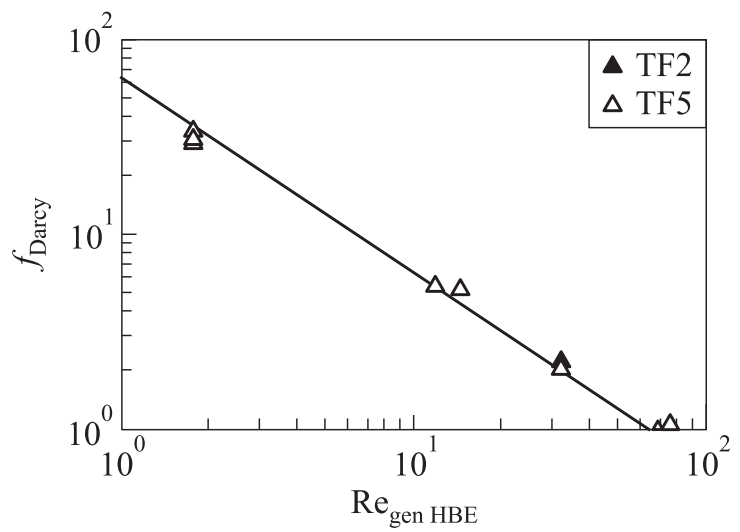

(a)

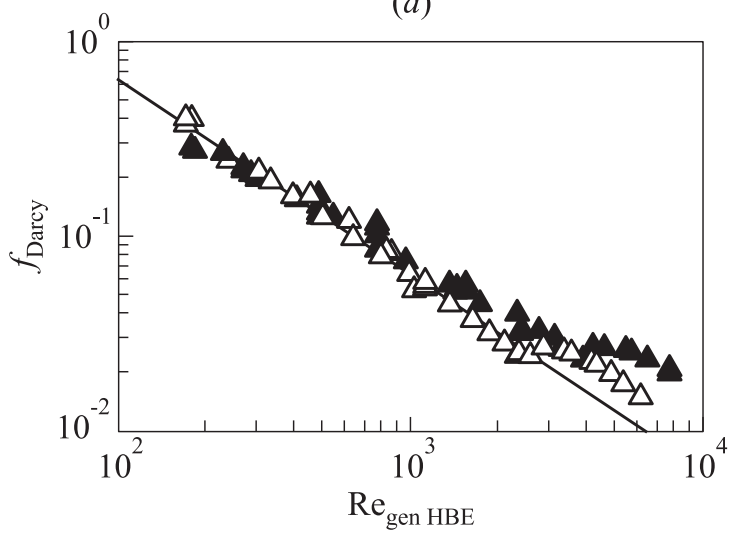

(b)

Figure 3 Friction factor vs. HBE-generalized low $(a)$ and high $(b)$ Reynolds number for liquid (TF2) and gelled (TF5 - trixatrol) propellants based on kerosene

numbers, an increase in the friction factor values in Fig. $2 c$ can be seen which will be discussed later.

Figures 3 and 4 show the friction factor values of the kerosene- and the paraffin-based fuels plotted against the HBE-generalized Reynolds number. The results are presented in separate diagrams for low and high Reynolds numbers. The values of the Darcy friction factor were calculated from Eq. (6) taking into account the experimentally determined pressure loss for a given flow rate and capillary geometry. The corresponding HBE-generalized Reynolds numbers for the given flow rate were calculated with Eq. (12) taking into account fluid properties and viscosity parameters. For the liquid kerosene and the kerosene-gel in Fig. 3, the relation $f_{\text {Darcy }}=64 /$ Re for laminar flow (in- 


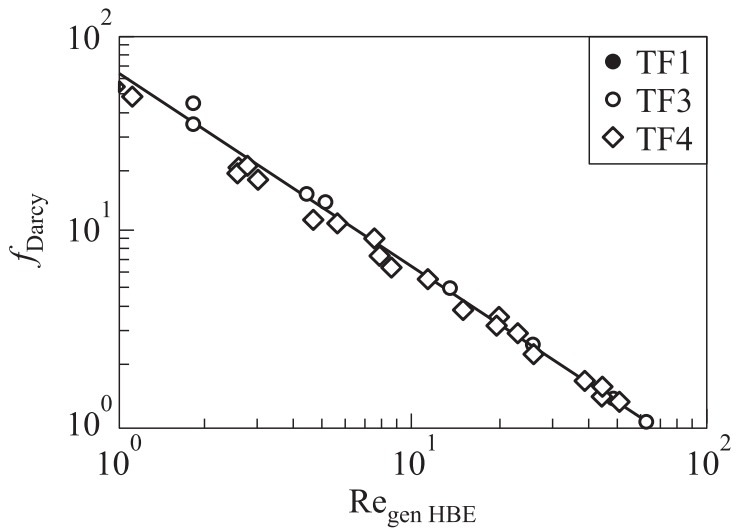

(a)

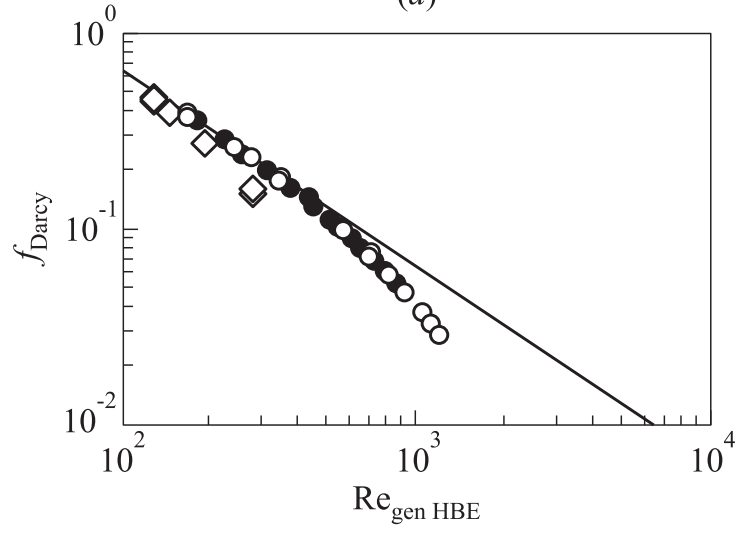

(b)

Figure 4 Friction factor vs. HBE-generalized low $(a)$ and high $(b)$ Reynolds number for liquid (TF1) and gelled (TF3 and TF4) propellants based on paraffin

dicated with the solid line) is successfully achieved up to Reynolds numbers of $\mathrm{Re}_{\text {gen } \mathrm{HBE}} \approx 1000$.

At higher Reynolds numbers, slightly lower values compared to the relation $f_{\text {Darcy }}=64 / \operatorname{Re}$ occur, before in the range between $2000<\operatorname{Re}_{\text {gen HBE }}<3000$ the experimental results show values significantly higher than the solid line. In view of the well-known results for Newtonian liquids and in view of the publications concerning the duct flow of non-Newtonian fluids (see, e.g., [3]), it is assumed that this increase is indicative of the transition from laminar to turbulent flow. It has to be mentioned that this transition was measured for both Newtonian liquid (kerosene) and non-Newtonian (kerosene-gel). There is still no definition for the critical HBE-generalized Reynolds number valid for such non-Newtonian 
HBE-fluids. Dodge and Metzner [3] and Ryan and Johnson [4] made investigations which, however, are only valid for pure PL fluids and cannot be applied to the fluids investigated in the present paper. Since the viscosity of the kerosenegel reaches the Newtonian plateau at high shear rates caused by high volumetric flow rates, it is assumed that the transition from laminar to turbulent flow conditions for the kerosene-gel could occur also near the critical Reynolds numbers of Newtonian liquids $\left(\operatorname{Re}_{\text {crit Newton }} \approx 2300\right)$.

For the liquid paraffin and the two paraffin-gels, the experimental results are plotted in Fig. 4. Due to the higher viscosity of the paraffin-based fluids compared to the kerosene-based fluids, lower Reynolds numbers are achieved. The data show a good agreement with the laminar relation (solid line) up to $\mathrm{Re}_{\text {gen HBE }} \approx 200$. The transition from laminar to turbulent flow could not be reached in the conducted experiments. The deviation from the solid line which was obtained for the kerosene-based fluids just before the laminar-to-turbulent transition seems to be significantly more pronounced for the paraffin-based fuels. There is no explanation yet for this deviation. Since the (Newtonian) liquid paraffin shows the same negative deviation from the solid line in Fig. $4 b$ as the paraffin-gels, it is assumed that this is not due to the non-Newtonian viscosity characteristic of the gels. All data are corrected with Bagley [14] and the non-Newtonian gels additionally with the Weissenberg-Rabinowitsch correction. However, obviously, there might be effects due to the very small capillary diameters and the inlet geometry which are more pronounced for high-viscosity fluids compared to low-viscosity fluids (e.g., vena contracta). Hence, further investigations are required.

\section{SUMMARY}

In the present paper, it was suggested to use the introduced HBE-generalized Reynolds number $\mathrm{Re}_{\text {gen HBE }}$ for characterizing the duct flow conditions of the investigated non-Newtonian gelled fluids. The Reynolds number is based on the HBE viscosity law. Experimental data of capillary rheometer measurements were used to verify, whether the general relation $f_{\text {Darcy }}=64 /$ Re between the Darcy friction factor and the Reynolds number is fulfilled in the laminar flow region. The results obtained with the introduced HBE-generalized Reynolds number were compared to the results obtained with the well-known definitions of Reynolds numbers for Newtonian and PL fluids. It was shown for the kerosenethixatrol-gel that the relation in the laminar flow region could only be fullfilled if the introduced HBE-generalized Reynolds number was applied to the experimental data. For Reynolds numbers between $2000<\operatorname{Re}_{\text {gen } \mathrm{HBE}}<3000$, a significant increase of the friction factor was obtained for the kerosene-based fluids. This increase was assumed to be an indication for the laminar-to-turbulent transition in the flow. However, further investigations are required for the verification of this 
assumption. For the paraffin-based fluids, a good agreement was obtained between the experimental data and the theoretical relation $f_{\text {Darcy }}=64 / \mathrm{Re}_{\text {gen HBE }}$ in the laminar flow region for low Reynolds numbers. For high Reynolds numbers, a significant negative deviation of the friction factor from the laminar relation $f_{\text {Darcy }}=64 / \operatorname{Re}_{\text {gen HBE }}$ was found. Since this effect was measured also for the liquid paraffin, it is assumed that it is not due to the non-Newtonian viscosity characteristic of the gels. For very high Reynolds numbers, there are obviously other fluid mechanical effects due to the use of very small capillary diameters which are more distinct for high-viscosity fluids compared to low-viscosity fluids.

\section{REFERENCES}

1. Brinker, C. J., and G. W. Scherer. 1990. Sol-gel science. Boston: Academic Press.

2. Ciezki, H. K., and B. Natan. 2005. An overview of investigations on gel fuels for ramjet applications. ISABE Paper No. 2005-1065.

3. Dodge, D. W., and A. B. Metzner. 1959. Turbulent flow of non-Newtonian systems. AIChE J. 5(2):198-204.

4. Ryan, N. W., and M. M. Johnson. 1959. Transition from laminar to turbulent flow in pipes. AIChE J. 5(4):433-35.

5. Mishra, P., and G. Tripathi. 1970. Transition from laminar to turbulent flow of purely viscous non-Newtonian fluids in tubes. Chem. Eng. Sci. 26(6):915-21.

6. Malin, M. R. 1998. Turbulent pipe flow of Herschel-Bulkley fluids. Int. Commun. Heat Mass Transfer 25(3):321-30.

7. Böhme. G. 2000. Strömungsmechanik nichtnewtonscher Fluide. Teubner Verlag, 2. Auflage, 2000.

8. Metzner, A. B., and J. C. Reed. 1955. Flow of non-Newtonian fluids - correlation of the laminar, transition and turbulent-flow regions. AIChE J. 1(4):434-40.

9. Madlener, K., and H. K. Ciezki. 2005. Analytical description of the flow behaviour of extended-Herschel-Bulkley fluids with regard to gel propellants. 36th Conference (International) of ICT \& 32nd Pyrotecnics Seminar (International). Karlsruhe, Germany.

10. Madlener, K., and H.K. Ciezki. 2005. Theoretical investigation of the flow behaviour of gelled fuels of the extended Herschel-Bulkley type. 1st European Conference for Aerospace Science (EUCASS). Moscow, Russia.

11. Hutter, K. 2002. Fluid- und Thermodynamik. Auflage: Springer Verlag.

12. Rabinowitsch, B. 1929. Über die Viskosität und Elastizität von Solen. Z. Phys. Chem. 145A.

13. Mooney, M. 1931. Explicit formulas for slip and fluidity. J. Rheol. 2(2):210-22.

14. Bagley, E. B. 1957. End corrections in the capillary flow of polyethylene. J. Appl. Phys. 28(5):624-27. 